\title{
Recurrent SPECC1L-NTRK fusions in pediatric sarcoma and brain tumors
}

\author{
Dong-Anh Khuong-Quang, 1,2,3 Lauren M. Brown, ${ }^{1,3,4}$ Marie Wong, ${ }^{1,5}$ \\ Chelsea Mayoh, ${ }^{1}$ Alexandra Sexton-Oates, ${ }^{3}$ Amit Kumar, ${ }^{1,6}$ Mark Pinese, ${ }^{1}$ \\ Sumanth Nagabushan, ${ }^{7}$ Loretta Lau, ${ }^{1,7}$ Louise E. Ludlow, ${ }^{3}$ Andrew J. Gifford, ${ }^{1,8}$ \\ Michael Rodriguez, ${ }^{8}$ Jayesh Desai, ${ }^{6,9}$ Stephen B. Fox, ${ }^{9,10}$ Michelle Haber, ${ }^{1}$ David \\ S. Ziegler, ${ }^{1,7}$ Jordan R. Hansford, ${ }^{2,3,4}$ Glenn M. Marshall, ${ }^{1,7}$ Mark J. Cowley, ${ }^{1,5}$ \\ and Paul G. Ekert 1,3,5,6
${ }^{1}$ Children's Cancer Institute, University of New South Wales, Randwick, 2031, Australia; ${ }^{2}$ Children's Cancer Centre, ${ }^{3}$ Murdoch Children's Research Institute, Royal Children's Hospital, Parkville, 3052, Australia; ${ }^{4}$ Department of Pediatrics, University of Melbourne, Parkville, 3052, Australia; ${ }^{5}$ School of Women's and Children's Health, UNSW Medicine, UNSW Sydney, Randwick, 2031, Australia; ${ }^{6}$ Peter MacCallum Cancer Centre, Melbourne, 3000, Australia; ${ }^{7}$ Kids Cancer Centre, Sydney Children's Hospital, Randwick, 2031, Australia; ${ }^{8}$ Department of Anatomical Pathology, Prince of Wales Hospital, Randwick, 2031, Australia; ${ }^{9}$ Sir Peter MacCallum Department of Oncology, University of Melbourne, 3000, Australia; ${ }^{10}$ Department of Pathology, Peter MacCallum Cancer Centre, Melbourne, 3000, Australia

Corresponding author: PEkert@ccia.org.au

(C) 2020 Khuong-Quang et al. This article is distributed under the terms of the Creative Commons Attribution-NonCommercial License, which permits reuse and redistribution, except for commercial purposes, provided that the original author and source are credited.

Ontology term: neoplasm of the central nervous system

Published by Cold Spring Harbor Laboratory Press

doi:10.1101/mcs.a005710
Abstract The identification of rearrangements driving expression of neurotrophic receptor tyrosine kinase (NTRK) family kinases in tumors has become critically important because of the availability of effective, specific inhibitor drugs. Whole-genome sequencing (WGS) combined with RNA sequencing (RNA-seq) can identify novel and recurrent expressed fusions. Here we describe three SPECC1L-NTRK fusions identified in two pediatric central nervous system cancers and an extracranial solid tumor using WGS and RNA-seq. These fusions arose either through a simple balanced rearrangement or in the context of a complex chromoplexy event. We cloned the SPECC1L-NTRK2 fusion directly from a patient sample and showed that enforced expression of this fusion is sufficient to promote cytokine-independent survival and proliferation. Cells transformed by SPECC1L-NTRK2 expression are sensitive to a TRK inhibitor drug. We report here that SPECC1L-NTRK fusions can arise in a range of pediatric cancers. Although WGS and RNA-seq are not required to detect NTRK fusions, these techniques may be of benefit when NTRK fusions are not suspected on clinical grounds or not identified by other methods.

[Supplemental material is available for this article.]

\section{INTRODUCTION}

Genomic and transcriptomic sequencing of pediatric cancers has broadened the spectrum of driver genetic alterations recognized across diverse tumor types. A subset of these drivers is targetable with novel therapies, which in some cases provide clinical options when none previously existed. The TRK family of receptors, encoded by the NTRK1, NTRK2, and NTRK3 genes, are proto-oncogenes regulating signaling pathways, including the MAPK and PI3K pathways, and play a critical role in neuronal development and differentiation. Fusions that link protein-protein interaction domains to the tyrosine kinase domain of each of the NTRK genes are recognized oncogenic drivers. NTRK fusions were first described in 
colorectal carcinoma (Martin-Zanca et al. 1986) and papillary thyroid carcinoma (Bongarzone et al. 1989) and later in a broad range of cancer types including pediatric cancers (Knezevich et al. 1998; Davis et al. 2018). NTRK fusions are strongly associated with particular rare pediatric tumor types, notably infantile fibrosarcoma (Knezevich et al. 1998), in which 70\%-90\% of cases harbor recurrent ETV6-NTRK3 fusions (Bourgeois et al. 2000; Sheng et al. 2001). NTRK fusions also characterize a subset of more common tumors, such as pediatric gliomas (Mackay et al. 2017; Guerreiro Stucklin et al. 2019; Clarke et al. 2020).

The clinical identification of NTRK fusions is a high priority because of the availability and clinical efficacy of TRK inhibitors (Drilon et al. 2018). The dramatic antitumoral activity of TRK inhibitors appears to be independent of the clinical features of the tumor in which it arises, including histological type, patient age, and genomic mechanisms generating the fusion (Drilon et al. 2018; Laetsch et al. 2018). Larotrectinib is only the second agent to be granted U.S. Federal Drug Administration (FDA) approval based on the presence of a molecular alteration, regardless of the type of cancer in which the mutation occurs, and a trial in previously untreated pediatric solid tumors is underway (COG ADVL1823-NCT03834961). To emphasize the importance of detecting a potential NTRK fusion, the European Society for Medical Oncology (ESMO) Translational Research and Precision Medicine Working Group has recommended testing for NTRK fusions in all advanced stage malignancies, particularly if standard testing has not identified clear driver mutations (Marchio et al. 2019).

Whole-genome sequencing (WGS) and whole-transcriptome RNA sequencing (RNAseq) offer an unbiased methodology to identify the presence of all expressed structural variants such as NTRK fusions. NTRK fusions, either recurrent, rare, or with unknown fusion partners can also be detected with a range of next-generation sequencing (NGS) approaches, including panels sequencing RNA after library preparation based on hybridization capture or anchored multiplex polymerase chain reaction (PCR) (Seager et al. 2019). More specific and sensitive genetic techniques including fluorescence in situ hybridization (FISH) and PCR-based assays are efficient and rapid but are limited to known fusions and risk missing unique or rare recurrent NTRK fusions. Immunohistochemical techniques detect TRK overexpression as a surrogate for the presence of a driver fusion but have limitations in specificity, particularly for NTRK3 fusions (Penault-Llorca et al. 2019).

Here, we report the genomic features of three instances of a recurrent NTRK fusion partner, SPECC1L, in diverse pediatric cancer subtypes. The mechanisms giving rise to these fusions vary from simple reciprocal translocations to very complex chromosomal events resembling chromoplexy. We have cloned and expressed the SPECC1L-NTRK2 fusion and show that it is sufficient to permit cytokine-independent survival and proliferation. These data emphasize the significant potential clinical benefit of performing WGS and RNA-seq to identify recurrent targetable lesions in difficult-to-treat tumors in which driver lesions have not been identified by conventional testing.

\section{RESULTS}

\section{Clinical Presentation}

Table 1 summarizes the clinical details of each of the three patients. Patient 1 presented with neurological symptoms of ataxia and intermittent headaches and was diagnosed at the age of $10 \mathrm{yr}$ with an unusual pineal low-grade neuroepithelial tumor. The tumor had two morphologically distinct components. One contained a moderate density of mildly pleomorphic, strongly GFAP-positive, Olig2- and CD34-negative glial cells with extensive psammomatous calcification. The other component was more cellular, containing plump spindle-shaped and large epithelioid cells with occasional perivascular pseudorosettes resembling astroblastomatous pseudorosette. Most of these cells were also strongly GFAP-positive, although some of 


\begin{tabular}{|c|c|c|c|}
\hline Gender & M & M & $\mathrm{F}$ \\
\hline Age & $10 \mathrm{yr}$ & $16 \mathrm{mo}$ & $11 \mathrm{mo}$ \\
\hline $\begin{array}{l}\text { Clinical } \\
\text { presentation }\end{array}$ & $\begin{array}{l}\text { Three weeks history of } \\
\text { dizziness, ataxia, and } \\
\text { intermittent headaches }\end{array}$ & $\begin{array}{l}\text { Epilepsy and focal } \\
\text { neurological deficit }\end{array}$ & $\begin{array}{l}\text { Failure to thrive, } \\
\text { developmental regression, } \\
\text { and intermittent fevers; } \\
\text { acute respiratory distress }\end{array}$ \\
\hline Diagnosis & $\begin{array}{l}\text { Low-grade } \\
\text { neuroepithelial tumor }\end{array}$ & Anaplastic astrocytoma & Infantile fibrosarcoma \\
\hline Primary tumor & Pineal & Left thalamus & Chest \\
\hline Staging & Localized & Localized & Localized \\
\hline Histopathology & $\begin{array}{l}\text { Low-grade } \\
\text { neuroepithelial tumor }\end{array}$ & Anaplastic astrocytoma & Congenital infantile sarcoma \\
\hline Ki67 staining & Ki67 2\%-3\% & Ki67 10\% & Ki67 10\% \\
\hline Pretreatment & Surgery alone & $\begin{array}{l}\text { Carboplatin-based infant } \\
\text { regimen (five cycles } \\
\text { before tumor } \\
\text { progression) }\end{array}$ & $\begin{array}{l}\text { VCR/Cyclo/ActD followed by } \\
\text { VCR }\end{array}$ \\
\hline $\begin{array}{l}\text { Other reportable } \\
\text { findings }\end{array}$ & - & $\begin{array}{l}\text { Homozygous loss of } \\
\text { CDKN2A/B }\end{array}$ & - \\
\hline Treatment & Surveillance & Larotrectinib & Larotrectinib \\
\hline Status & SD 22 mo postsurgery & $\begin{array}{l}\text { SD on larotrectinib for } \\
33 \text { mo }\end{array}$ & $\begin{array}{l}\text { CR postsurgery after } 22 \text { mo } \\
\text { on larotrectinib }\end{array}$ \\
\hline
\end{tabular}

(M) Male, (F) female, (VCR) vincristine, (Cyclo) cyclophosphamide, (ActD) dactinomycin, (FC) fold change, (SD) stable disease, (CR) complete remission.

the epithelioid cells did not stain. The epithelioid cells were strongly CD34-immunoreactive. Both components were S100- and CD56-positive and did not stain for neurofilament protein, synaptophysin, chromogranin, IDH1 R132H, H3K27M, or BRAF ${ }^{\mathrm{V} 600 \mathrm{E}}$. No mitotic figures were identified, and the Ki67 labeling index was low (2\%-3\%). No necrosis or microvascular proliferation was identified. The tumor could not be classified using the current (2016) WHO classification and consequently cannot be given a WHO grade, although there were no high-grade features. The tumor was partially resected, and third ventriculostomy performed. As part of an ongoing research project, DNA methylation profiling using the Illumina Infinium HumanMethylation 850k BeadChip and DNA panel sequencing as previously described (Sahm et al. 2016) were performed. Although the tumor did not match with any reference class of central nervous system tumor (highest score for the methylation class family lowgrade glioma, subclass ganglioglioma with a score of 0.71) using the DNA methylationbased classifier developed by the German Cancer Research Centre (DKFZ) (Capper et al. 2018), the DNA panel sequencing suggested the possibility of an NTRK fusion. NTRK fusions are not currently tested for as a part of standard-of-care clinical diagnostics for this type for tumor in Australia. However, research-based WGS and RNA-seq identified a SPECC1LNTRK2 fusion. Currently the patient is not receiving any treatment and has stable residual disease without any sign of progression, 20 mo after the surgery.

Patient 2 presented with seizures and focal neurological deficit and was diagnosed at the age of 16 mo with an unresectable thalamic anaplastic astrocytoma (WHO grade III). The tumor presented dense and uniform cells with marked atypia, mitotic figures. No necrosis or microvascular proliferation was seen. The Ki-67 was 10\%, and the positive staining for WT-1, S100, and restricted GFAP expression as well as negative synaptophysin staining are mostly in keeping with anaplastic astrocytoma. This tumor was negative for H3.3, 
$\mathrm{H} 3.3 \mathrm{~B}$, and $\mathrm{H} 3.1 \mathrm{~K} 27$ and $\mathrm{G} 34$ mutations. No BRAF fusions or BRAF mutations were identified. The tumor progressed despite treatment with carboplatin-based chemotherapy as previously reported (Fouladi et al. 2009). RNA-seq, performed as an adjunct to a clinical trial of targeted amplicon DNA sequencing (iPredict flagship, Melbourne Genomics Health Alliance [MGHA]), identified the SPECC1L-NTRK2 fusion, which was confirmed by subsequent WGS. The patient was commenced on larotrectinib following enrolment onto a phase 1 clinical trial (NCT02637687), with no further disease progression and continues on therapy with stable disease for 33 mo.

Patient 3 was diagnosed at the age of 11 mo with a large congenital infantile fibrosarcoma of the chest wall compressing the lungs and airways. The tumor had a varied appearance. In areas (demonstrated later in Fig. 2), the tumor consisted of intersecting fascicles of densely packed spindle cells with scant eosinophilic to amphophilic cytoplasm. Elsewhere the tumor contained fibrocollagenous stroma infiltrated with tumor cells that had more eosinophilic to vacuolated cytoplasm. The patient had no response to conventional chemotherapy and required intubation and ventilation with increasingly difficult ventilatory requirements. The presence of an NTRK fusion was suspected based on the histological diagnosis. ETV6 break-apart FISH was performed on a biopsy specimen to identify an ETV6-NTRK3 fusion, and the result was negative. A SPECC1L-NTRK3 fusion was identified by WGS and RNAseq. The patient was commenced on larotrectinib with excellent clinical response and near-complete resolution of disease on radiological imaging.

\section{Genomic Analyses}

The variant table (Table 2) shows the details of the SPECC1L-NTRK fusions, including the genomic breakpoints and the sequencing platforms used to identify the fusions. The range of somatic single-nucleotide variants (SNVs), copy-number variants (CNVs), and structural variants (SVs) are shown in Supplemental Table 1. All three samples were sequenced as part of studies in which somatic and germline sequencing are performed to identify potential clinically actionable variants (Wong et al. 2020). In each patient, the NTRK fusion was identified as the highest priority actionable target. The SNV tumor mutation burden was $<1$ mutation/Mb in each instance.

The somatic genomes of the SPECC1L-NTRK fusions are summarized in the Circos plots shown in Supplemental Figure 1. In all three patients, the NTRK fusions are also annotated as potentially linked to the ADORA2A gene (ENST00000337539), which is located at the same locus as SPECC1L and a SPECC1L-ADORA2A readthrough transcript is included in the reference genome. However, the expressed fusions include only SPECC1L (Fig. 1A-E). The tumor from Patient 1 harbored multiple whole-chromosomal gains in contrast with the tumor from Patient 2, which was less aneuploid but harbored a biallelic loss of CDKN2A/B (Chr 9). The SPECC1L breakpoints in Patients 1 and 2 also differed, in intron 12 and intron 11, respectively (Fig. 1A,B); however, in each instance the fusion remained in frame. The SPECC1L-NTRK3 fusion (Patient 3) arose from a complex chromoplexy event involving multiple structural variants and copy-number aberrations on Chromosome 15 (Supplemental Fig. 1D). An inversion involving exons 14 to 19 of NTRK3, encoding the tyrosine kinase domain, resulted in an in-frame fusion to the first nine exons of SPECC1L (Fig. 1C-E). In each case, although the SPECC1L breakpoint differs, the SMC (structural maintenance of chromosomes) domain of SPECC1L, which is involved in heterodimerization of SMC protein family members and contains an extended coiled-coil region, is retained in-frame with the tyrosine kinase domains of NTRK2 or NTRK3. Immunohistochemistry confirmed the expression of TRK in all three tumors (Fig. 2).

LINX, which clusters together contemporaneous individual copy-number changes and structural variant calls, was used to provide a visualization of the structure of derivative 


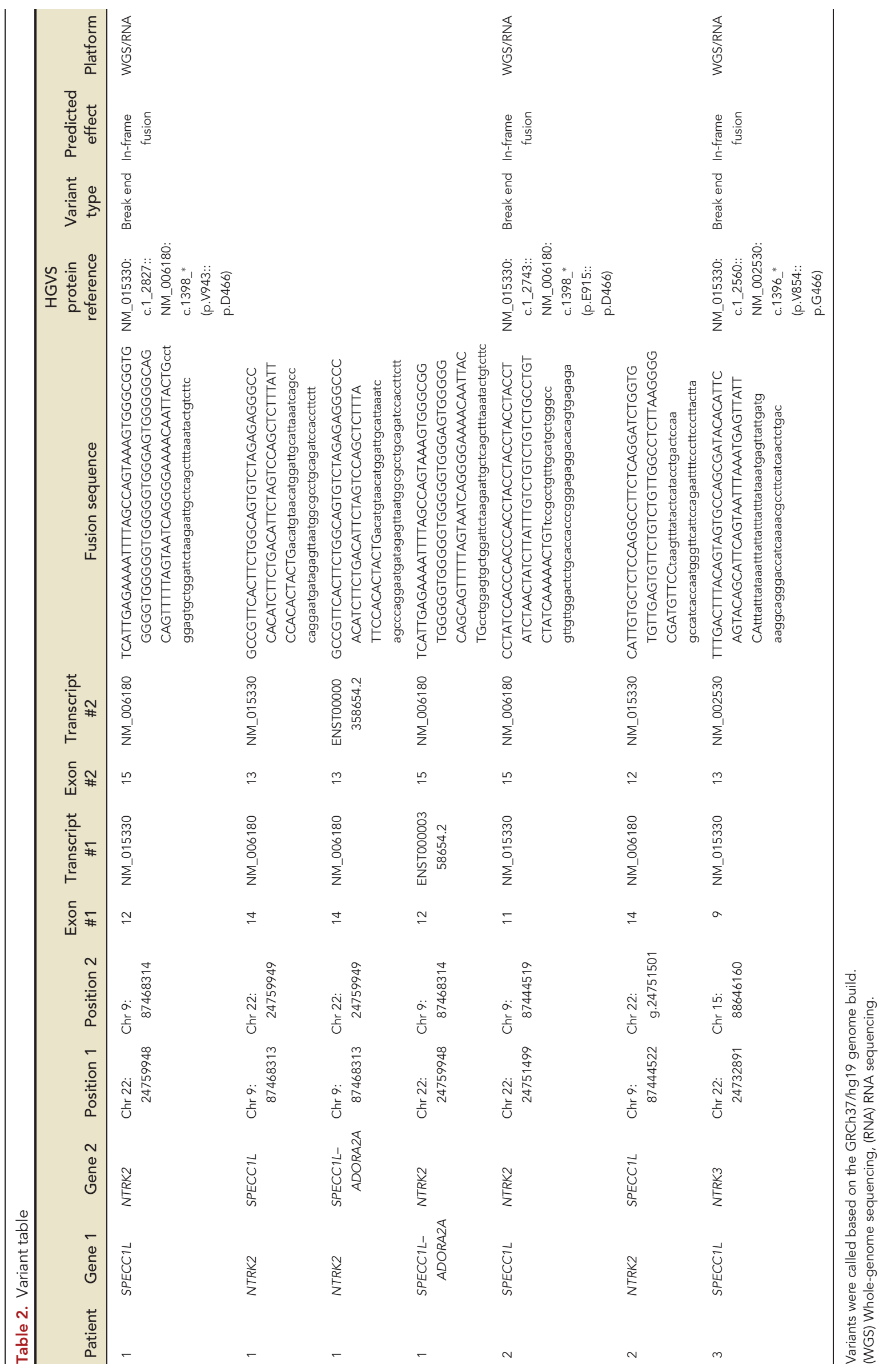




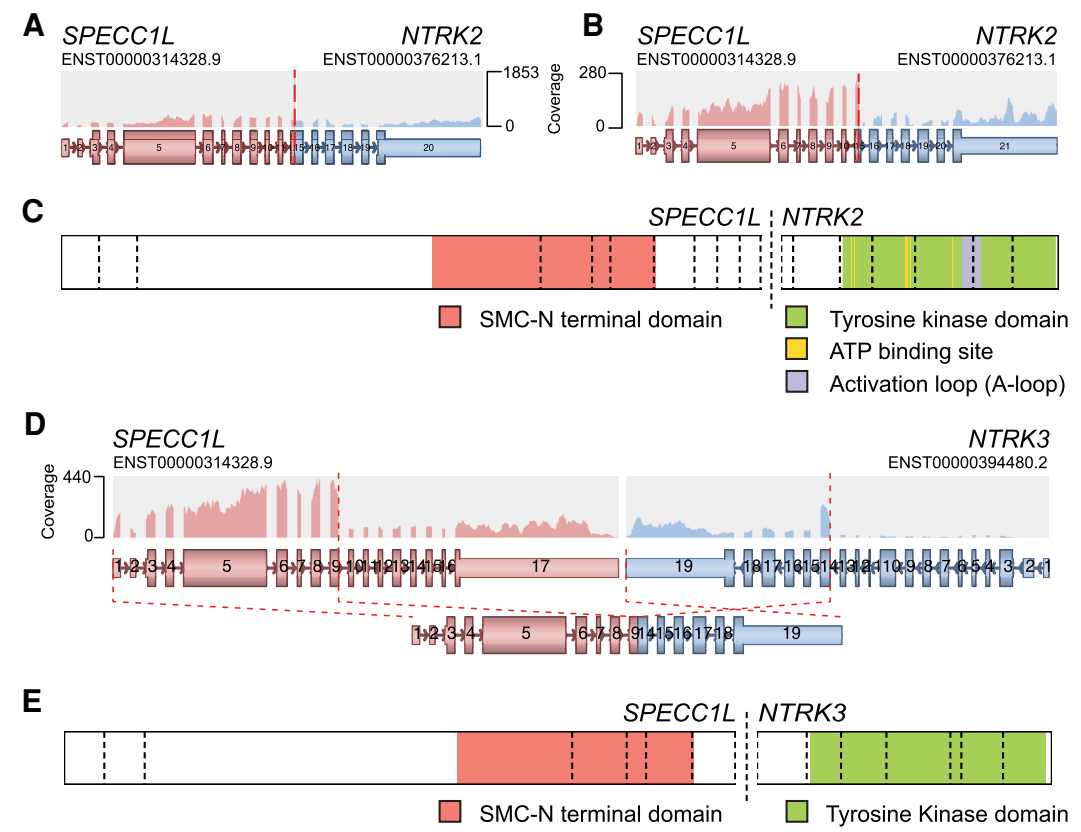

Figure 1. SPECC1L-NTRK fusions arising in an infantile fibrosarcoma and two pediatric central nervous system tumors. $(A, B)$ SPECC1L-NTRK2 fusions identified in Patients 1 and 2 from transcriptomic data. RNAseq data was analyzed using the Arriba algorithm (see Methods section), and the visualizations are modified from the Arriba output. The SPECC1L and NTRK2 Ensembl reference transcripts are indicated above the read coverage in each gene. The breakpoints are indicated by the dashed red line. The structure of the inframe fusion and the involved exons is shown. (C) Representation of the SPECC1L-NTRK2 fusion generated using ProteinPaint (Zhou et al. 2016), showing the conserved protein domains in the fusion protein. The key for protein domains retained in the fusion protein is shown below the schematic. (D) Arriba analysis of Patient 3 and the SPECC1L-NTRK3 fusion. Note the inversion of the exons encoding the tyrosine kinase domain of NTRK3 that results in the in-frame fusion. (E) Representation of the SPECC1L-NTRK3 fusion generated using ProteinPaint, showing the conserved protein domains in the fusion protein.

chromosomes (Fig. 3; Cameron et al. 2019). Patient 1 had a duplication encompassing the NTRK2 tyrosine kinase domain linked to the SPECC1L SMC domain (copy number =2). The derivative Chr 22 included small regions with varying copy-number states, but that did not involve SPECC1L or NTRK2 (Fig. 3B). The fusion in Patient 2 is a simple reciprocal translocation (Fig. 3C). The SPECC1L-NTRK3 fusion has arisen from a much more complex event, with a derivative chromosome including break ends in Chr 15, Chr 22, and Chr X (Fig. 3D). The predicted fusion is flanked by more than 70 breakpoints associated with several copy-number states, a pattern consistent with chromoplexy (for review, see Shen 2013). The very complex structural variants in Chr 15 of Patient 3 were the primary genomic feature of this tumor. The expressed NTRK3 fusion is the likely mechanism favoring selection of this chromoplexy event in this tumor.

We additionally performed targeted sequencing (TSO500 panel-Tru-Sight Oncology 500 gene panel, Illumina) on formalin-fixed paraffin-embedded (FFPE) tissue from a resected sample from Patient 3 after 22 mo of larotrectinib therapy. No known resistance mutations in NTRK3 were found (Cocco et al. 2018), but evidence of reads spanning the original fusion breakpoint was identified, confirming persistent expression of the SPECC1L-NTRK3 fusion in residual tumor tissue (Supplemental Fig. 2). 


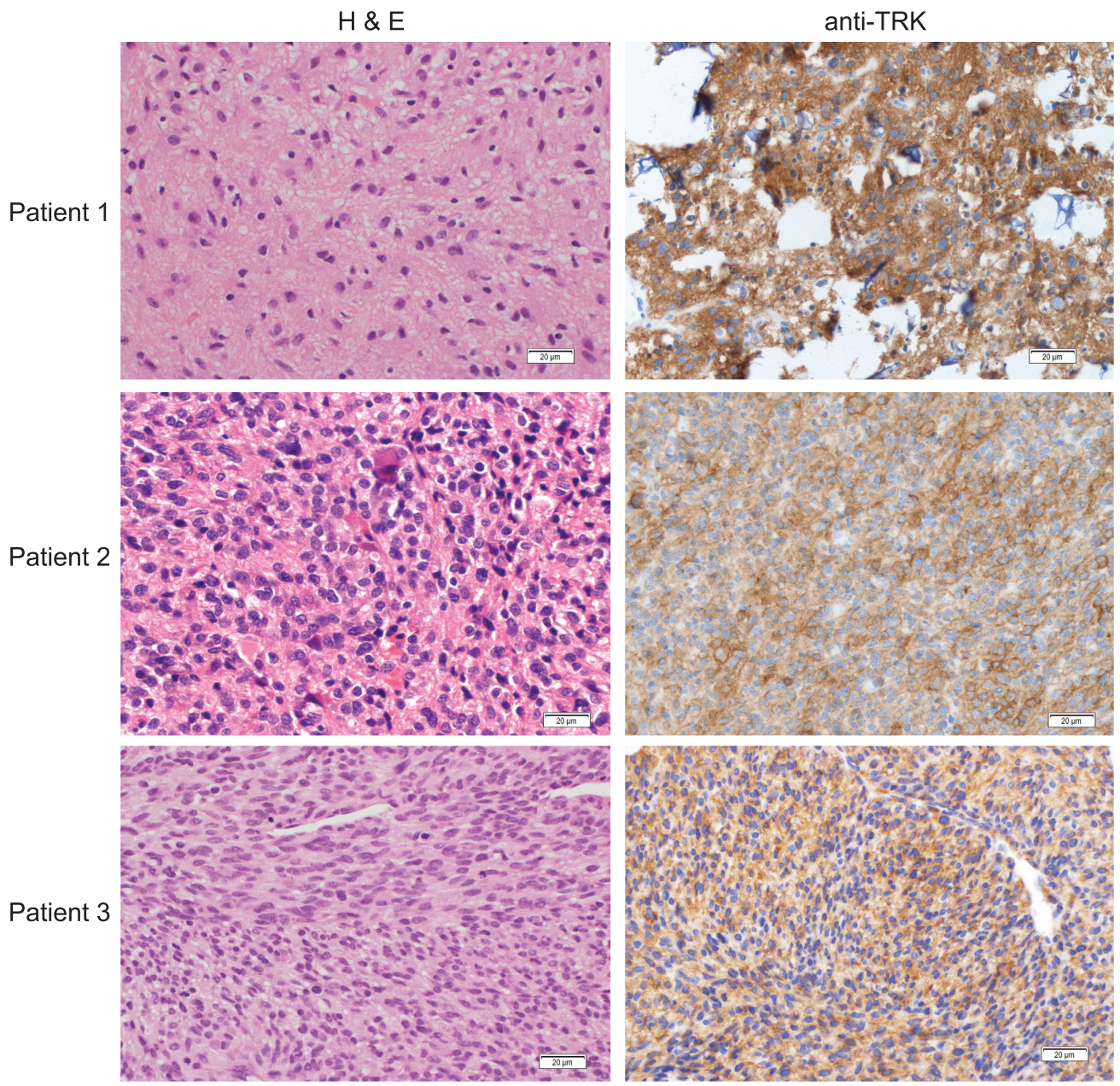

Figure 2. Immunohistochemistry of SPECC1L-NTRK fusion positive tumors shows high TRK expression. Hematoxylin and eosin staining and anti-TRK staining are shown for each of the patient tumor samples. Nonneoplastic cells served as internal negative control for the TRK antibody, and TRK expression was high for Patient 1, Patient 2, and Patient 3. Patient 1 presented with an unusual low-grade neuroepithelial tumor, Patient 2 with an anaplastic astrocytoma, and Patient 3 with an infantile fibrosarcoma. Further histologic description is detailed in the Clinical Presentation section in the main text.

\section{Functional Analyses}

We cloned the SPECC1L-NTRK2 fusion directly from cDNA derived from tumor RNA from the Patient 2 sample. There were two potential start sites that permitted transcription of an in-frame construct. The first was a full-length (FL SPECC1L-NTRK2) version of the fusion (transcription from the canonical start site of SPECC1L). The second was a truncated form (T SPECC1L-NTRK2) predicted to start from SPECC1L amino acid coding position 667 (ENST00000314228.9). The full-length form was the dominant transcript and we could not exclude the possibility that the truncated form was a PCR artifact. Both transcripts were expressed in interleukin-3 (IL-3)-dependent Ba/F3 cells under the regulation of a doxycycline-inducible promotor (Fig. 4A). Upon doxycycline addition, FL and T SPECC1L-NTRK2 fusion proteins were detected at their predicted sizes, 142 and $69 \mathrm{kDa}$, respectively, using a pan-Trk antibody (Fig. 4A). When IL-3 was removed from cultures, we observed increased expression of both fusions, likely the result of selection in favor of cells expressing 
A

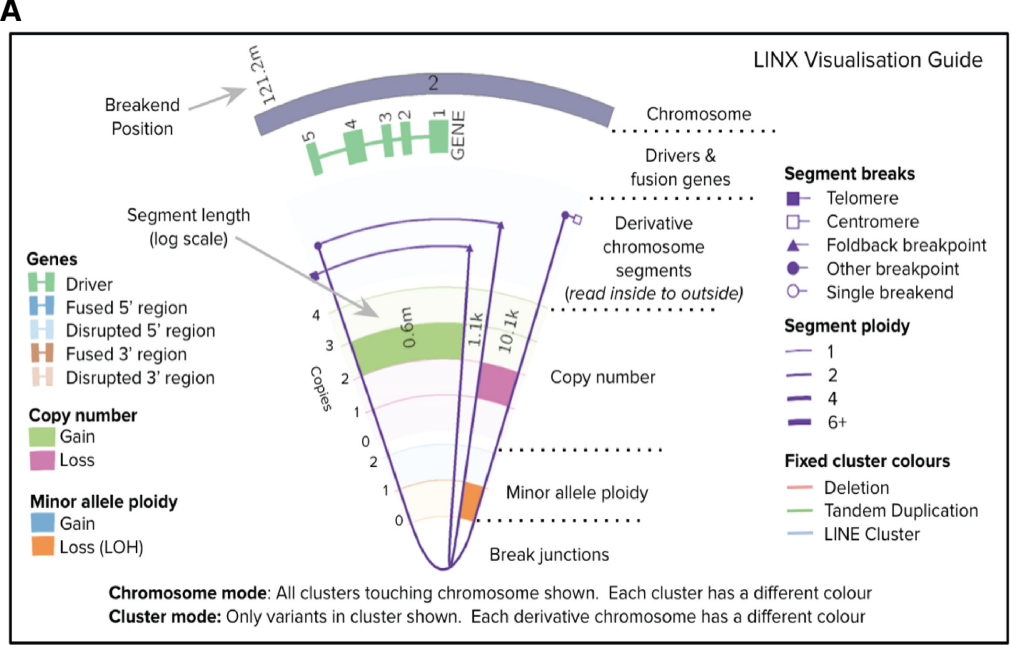
Cluster mode: Only variants in cluster shown. Each derivative chromosome has a different colour
B

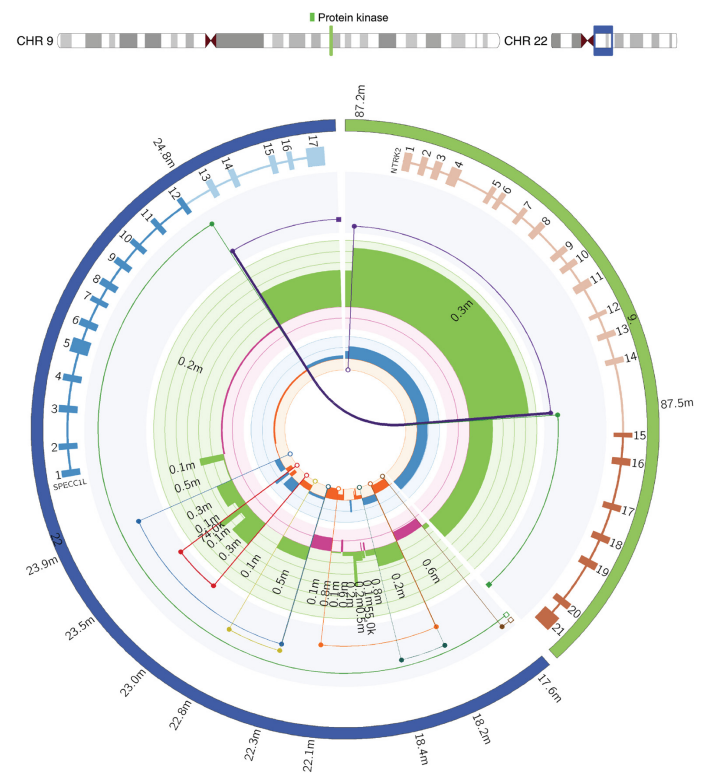

C CHR 9

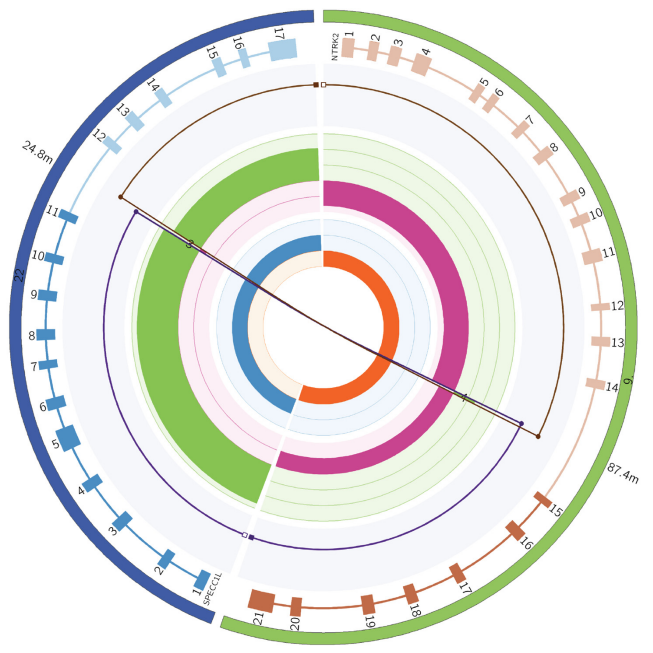

D

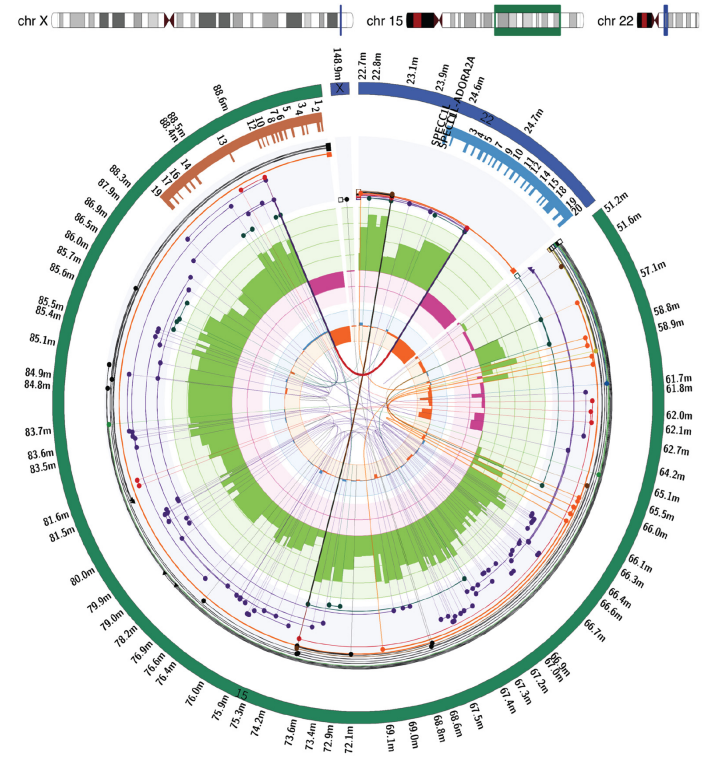

Figure 3. LINX plots of the derivative chromosomes encoding each of the NTRK fusions. (A) Key for the interpretation of the LINX plots. (Reproduced with permission from Cameron et al. 2019.) (B) LINX plot of Patient 1. This shows the fusion arising in association with a duplication event of NTRK2 with linked break ends juxtaposing the tyrosine kinase domain with SPECC1L SMC domain on Chromosome 22. Other linked segments with variable copy-alterations in Chromosome 15 are part of the derivative chromosome, but not associated with the fusion. (C) The NTRK2 fusion in Patient 2 is a simple reciprocal translocation associated with a copynumber gain of SPECC1L and loss of one copy of the region encoding the tyrosine kinase domain of NTRK2 (exons 15-21) and homozygous deletion of exon 1-14 of NTRK2. (D) The NTRK3 fusion arises from a complex structural event resembling chromoplexy, with multiple copy-number states and more than 70 breakpoints in the derivative chromosome. The regions of the NTRK3 and SPECC1L genes not involved in the fusion event are present as single copies only, having undergone a deletion event. 

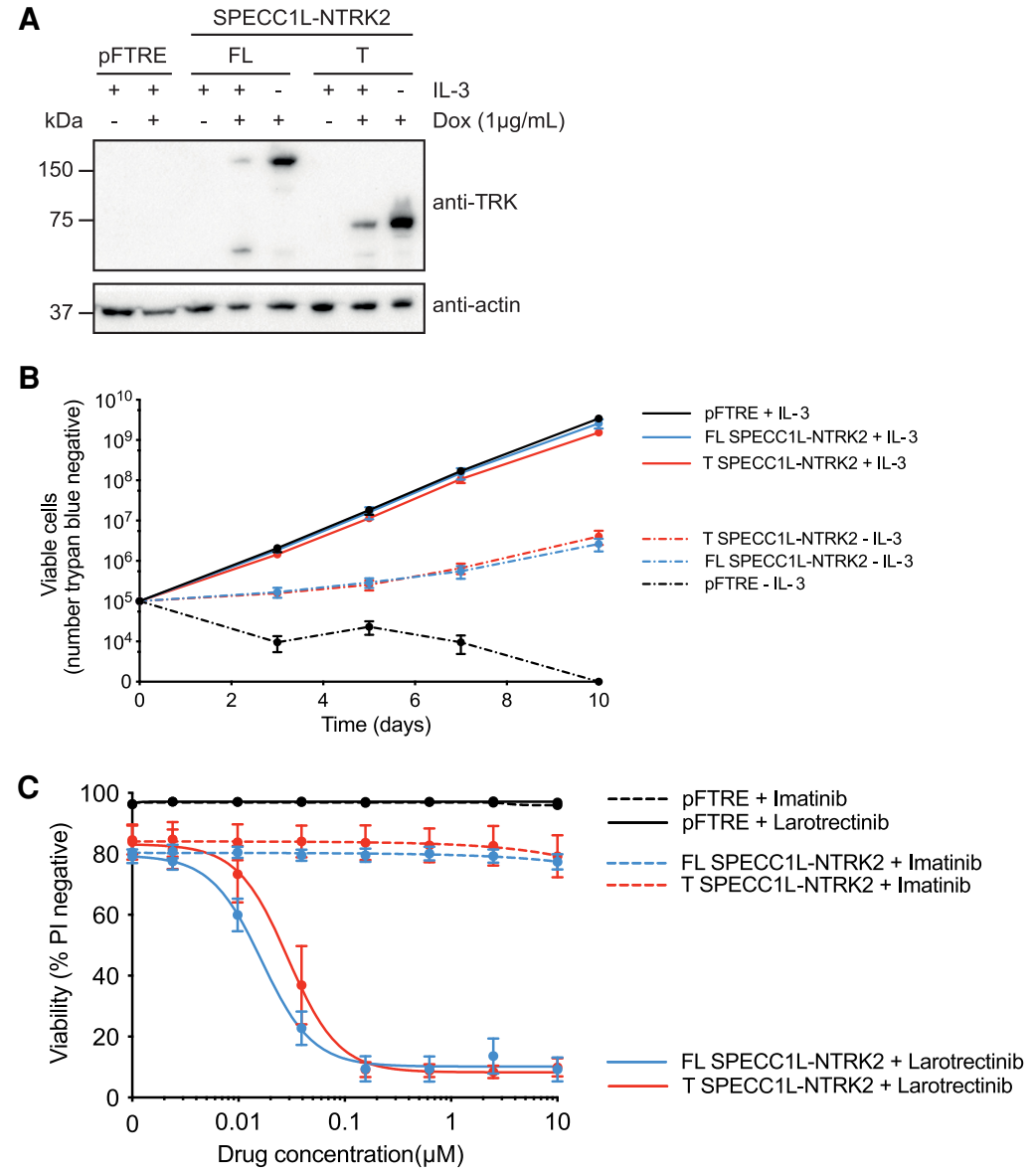

Figure 4. SPECC1L-NTRK2 fusions transform Ba/F3 cells to cytokine independence and these cells are sensitive to treatment with larotrectinib. (A) Western blot analysis of $B a / F 3$ cells expressing full-length (FL) and truncated (T) SPECC1L-NTRK2 fusions and empty vector controls ( $p F T R E)$. Cells were maintained in the presence (+) or absence (-) of IL-3, and fusion expression was driven by the addition of doxycycline (Dox) to cultures. Western blot probed with anti-TRK and anti- $\beta$-actin (anti-actin) was used as a loading control. (B) Viability analysis of doxycycline induced Ba/F3 cells seeded in the presence (solid line) and absence (dashed line) of IL3. Number of viable cells, measured by trypan blue exclusion, is shown on the $y$-axis, and time in days from seeding in the presence or absence of IL-3 is shown on the $x$-axis. (Data presented as mean $\pm S E M, n=4$.) (C) Viability analysis of SPECC1L-NTRK2 transformed Ba/F3 cells following TKI treatment. Ba/F3 cells transformed with FL SPECC1L-NTRK2 (blue line) or T SPECC1L-NTRK2 (red line), and empty vector controls (pFTRE, black line) were treated with a dose titration of imatinib (dashed line) or larotrectinib (solid line) for 48 h. Graph shows viability (\%) on the $y$-axis, determined by flow cytometry and PI exclusion, and the $x$-axis depicts increasing drug concentration in $\mu \mathrm{M}$. (Data presented as Mean $\pm \mathrm{SEM}, n=4$.)

higher levels of the fusion, and apoptosis of cells not expressing or expressing low levels of the fusion.

We then cultured the fusion-expressing cells and empty vector controls in the presence or absence of IL-3 over a 10-d time course (Fig. 4B), to assess the capacity of SPECC1 L-NTRK2 to promote long-term IL-3-independent proliferation in Ba/F3 cells. The number of FL and T SPECC1L-NTRK2-expressing viable cells increased over time in the absence of IL-3. In contrast, empty vector (pFTRE) control cells were dead by day 10 of IL-3 withdrawal (Fig. 4B). These data show that both the full-length and truncated SPECC1L-NTRK2 fusion blocks IL3 withdrawal-induced apoptosis and drives IL-3 independent proliferation in Ba/F3 cells. 
Following the generation of stably transformed Ba/F3 cell lines with SPECC1L-NTRK2 fusions, we next assessed the sensitivity of fusion expressing cells to a TRK inhibitor, larotrectinib. Control cells (maintained in IL-3) remained viable at all doses of larotrectinib and imatinib (tyrosine kinase inhibitor specific against ABL1, c-KIT, and PDGFR). FL SPECC1L-NTRK2- and T SPECC1L-NTRK2-expressing Ba/F3 cells, in which fusion expression is required to maintain viability in the absence of IL-3, died when exposed to a seven-point dose titration of larotrectinib but not imatinib (Fig. 4C). These data demonstrate that Ba/F3 cells transformed by FL SPECC1L-NTRK2 and T SPECC1L-NTRK2 fusions are specifically sensitive to TRK inhibitor treatment.

\section{DISCUSSION}

We report here three examples of recurrent NTRK fusions identified in three pediatric patients with three different histological diagnoses. This fusion is not detectable by routine FISH and highlights the role of genome-wide approaches like WGS and RNA-seq, in identifying diagnostic and therapeutic targets. The SPECC1L-NTRK2 fusion has been reported recently in pediatric glioma with anaplastic features (Torre et al. 2020) and in pediatric mixed neuronal glial tumor (Surrey et al. 2019), whereas the SPECC1L-NTRK3 fusion has been seen in undifferentiated sarcomas of the uterus (Gatalica et al. 2019; Hodgson et al. 2020; Rabban et al. 2020) and in mesenchymal tumors of the gastrointestinal tract (Atiq et al. 2020). SPECC1L is a gene encoding a coiled-coil domain protein located on 22q11.23. Germline loss-of-function mutations in SPECC1L have been identified in patients with oblique facial clefts (Saadi et al. 2011). Several recurrent partner genes have been reported to fuse with SPECC1L, including RET, which leads to constitutive RET kinase activation in papillary thyroid carcinomas (Cancer Genome Atlas Research Network 2014; Stransky et al. 2014), MET in lung adenocarcinoma (Nelson et al. 2019), and ALK in epithelioid fibrous histiocytoma (Dickson et al. 2018).

The SPECC1L SMC coiled-coil domains are retained in each of the NTRK fusion genes. Coiled-coil domains have been shown to play a role in activation of receptor tyrosine kinases by promoting dimerization and transphosphorylation. This was first shown for MET receptor (Rodrigues and Park 1993) and the BCR-ABL fusion protein (McWhirter et al. 1993), but evidence has since been extended to other oncoproteins including TRK fusions (Cocco et al. 2018). For many NTRK fusions, the fusion partner mediates activation of the receptor via dimerization, either through the addition of one of three known dimerization domainscoiled-coil, zinc finger, or WD domains-to the kinase domain of TRK or through alternate mechanisms (Cocco et al. 2018). We confirmed the oncogenic properties of the SPECC1L-NTRK2 fusion in vitro, where transformed Ba/F3 cells gained the capacity to grow independently of IL-3 and were specifically sensitive to larotrectinib. However, whereas $\mathrm{Ba} / \mathrm{F} 3$ is a gold-standard cell line model for exploring kinase-activating oncogenes in vitro (Roberts et al. 2014) and is a suitable model for first-pass assessment of the transforming capacity of this fusion, future work exploring the molecular mechanisms of oncogenesis by SPECC1L-NTRK2 will require the modeling of this fusion in the same cellular context in which SPECC1L-NTRK2 positive tumors arise.

In addition to the canonical ETV6-NTRK3 fusion, novel NTRK fusions have been identified in an increasing number of tumor types, both in children and adults. This includes pediatric soft-tissue sarcomas (Pavlick et al. 2017; Davis et al. 2018) and gliomas as reported here and elsewhere (Mackay et al. 2017; Guerreiro Stucklin et al. 2019; Clarke et al. 2020). Given the clinical implications for patients, it is now critical to identify these fusions at diagnosis, as TRK inhibitors are increasingly being integrated into patient treatments. 
Although pan-TRK immunochemistry, which detects TRK overexpression as a surrogate for the presence of NTRK fusion, is a highly sensitive tool to identify NTRK fusions at low cost and with a fast turnaround time, there are caveats that apply to this technique (Marchio et al. 2019). Most notably, the specificity is limited in tissues with physiological TRK protein expression, such as in the nervous system and smooth muscle (Murphy et al. 2017). For example, diffuse immunoreactivity is observed in $8 \%$ of spindle cell tumors without NTRK fusions (Hung et al. 2018). Moreover, the presence of an NTRK fusion may be associated with relatively weak pan-TRK staining (for review, see Marchio et al. 2019). Pan-TRK IHC also has limited specificity particularly for NTRK3 fusions (Penault-Llorca et al. 2019). Therefore, confirmation of the fusion by a second assay is widely recommended (Murphy et al. 2017; Marchio et al. 2019). The tumor resected after 22 mo of treatment with larotrectinib from Patient 3 did not stain at all with pan-TRK antibody (data not shown), despite the presence of the fusion in residual tumor. One explanation is that the acid decalcification step used prior to fixation and staining abolishes antibody detection of the epitope in fixed material.

Break-apart FISH is a cost-effective and well-recognized method to detect recurrent gene fusions. Because of the high prevalence of ETV6-NTRK fusions, an ETV6 probe has typically been used, despite a high false negative rate of up to $36 \%$ (Davis et al. 2018). It also fails to identify the NTRK partner gene. Therefore, NTRK break-apart FISH has been developed with a separate probe required for each of the three NTRK genes, making the test increasingly labor-intensive. Such tests are mostly recommended in the context of a tumor type with frequent NTRK fusion (Marchio et al. 2019) and do not exclude further testing if the result is negative. In the case of Patient 3, the ETV6 break-apart FISH testing failed to identify the NTRK fusion.

NGS is the method with the highest specificity and sensitivity to detect gene fusions compared with other testing methods; however, access may be limited because of the availability of the technique and its associated cost. Although DNA-based NGS panels are widely used in cancer, the detection of the fusion will depend on the platform used given that NTRK2 and NTRK3 genes have large introns that are typically inadequately sequenced and therefore difficult to analyze. To illustrate this difficulty, the NTRK2 fusion reported in Patient 2 was not detected by the custom targeted amplicon DNA sequencing performed, as neither the NTRK2 intron or the SPECC1L exons and introns involved in the fusion were included in the gene panel design. However, the fusion was detected by targeted panel sequencing in relapse material from Patient 3. An RNA-based NGS panel based on hybridization capture or anchored multiplex RT-PCR allows for detection of unknown partners, and both methods are currently used in the clinical setting (Jennings et al. 2017; Hsiao et al. 2019). In conclusion, we report three cases of recurrent SPECC1L-NTRK fusions in diverse pediatric malignancies. The fusions were definitively identified by high-throughput sequencing approaches that combined WGS and RNA-seq. WGS and RNA-seq are a highly effective and unbiased combination to identify these and other fusions.

\section{METHODS}

\section{Whole-Genome Sequencing}

For primary tumor tissue, DNA was extracted from fresh-frozen tissues using the AllPrep DNA/RNA/Protein Mini Kit (QIAGEN 8004) according to the manufacturer's instructions. WGS to intended coverage depths of $30 \times$ (normal tissue) and $90 \times$ (tumor tissue) was performed using Illumina's HiSeq X platform. Library preparation was performed using the TruSeq Nano DNA HT or Kapa HyperPrep PCR-free kit following the manufacturer's instructions. Details of sequencing coverage are presented in Supplemental Table 3. 
WGS data was processed using the in house refynr pipeline, utilizing DNAnexus (www .dnanexus.com), a cloud-based genomic analysis platform. Paired-end short reads were aligned to the hs37d5 reference genome, using BWA-MEM (v0l7.10-r789; Li and Durbin 2009) and duplicate reads marked and data from multiple lanes merged using Novosort (v1.03.01; default settings), followed by GATK IndelRealigner (v3.3; Van der Auwera et al. 2013). Germline SNVs and short indels (<50-bp) were identified using GATK HaplotypeCaller, GenotypeVCFs, and VQSR (v3.3; Van der Auwera et al. 2013), annotated with VEP (v87; McLaren et al. 2016), converted into a database using GEMINI (v0.11.0; Paila et al. 2013), and imported into Seave (Gayevskiy et al. 2019) for filtration and prioritization. Somatic SNVs and short (<50-bp) indels were identified using Strelka (v2.0.17; Saunders et al. 2012) and filtered using these criteria: QSS $\geq 10$ or QSI $\geq 10 ;$ NT = ref; from Chromosomes 1-22,X,Y; in at most 3 (of 2570) individuals in the similarly processed MGRB cohort (Pinese et al. 2020); if not in the platinum genome's high-confidence region, then with $\mathrm{VAF}>0.1$ and $\mathrm{QSS}>20$ or $\mathrm{QSI}>20$; VAF*OS $\geq 1.3$; Normal AF $>0$ and Tumor AF < $3^{*}$ normalAF; or in a curated hotspot white list. Somatic variants were annotated using SnpEff (v4_3t; Cingolani et al. 2012) and imported into in house Glooee platform for filtration and prioritization. Tumor purity and somatic CNV were assessed using PURPLE (v2.39, (Cameron et al. 2019). Somatic structural variants were identified using GRIDSS (v2.7.2), and derivative chromosomes were analyzed and visualized using LINX (v1.7; Cameron et al. 2019) using default settings.

\section{Whole-Transcriptome Data Analysis}

Whole-transcriptome RNA-seq was performed with TruSeq stranded mRNA preparation kit. Libraries were pooled and sequencing runs were performed in paired-end mode using either the Illumina HiSeq 4000 platform or NextSeq generating 40M reads per sample, respectively. Illumina paired-end RNA-seq data was aligned to the human genome assembly (build hg19) using STAR (version 2.5) 2-pass method with quantMode parameters set to TranscriptomeSAM for alignments translated into transcript coordinates. Alignments are sorted with SAMTools (version 1.3.1), duplicates marked with Picard Tools (version 2.4.1), reads are split and trimmed, and mapping qualities reassigned with Genome Analysis Toolkit (version 3.6) using methods SplitNCigarReads and ReassignOneMappingQuality, respectively. Raw gene counts, TPM, FPKM, and isoform expression values were calculated using RSEM (version 1.2.31) command rsem-calculate-expression. Fusions were identified using three methods, STAR-Fusion (version 1.3.1), JAFFA (version 1.09), and arriba (version 1.1.0). All fusions were reported as either high, medium, or low confidence, whether they were in-frame or not, and the type of rearrangement.

\section{TSO500 DNA/RNA Panel: Bioinformatic Analysis of TSO500}

DNA and RNA were extracted from FFPE blocks using standard protocols at the KCCG Cancer Diagnostics laboratory. DNA paired-end reads were processed using the Illumina TruSight Oncology 500 Local App (v1.0) Docker container, run on the DNAnexus platform using default options. RNA paired-end reads (14M reads) were aligned to hg19 using STAR (v2.5) 2-pass method, duplicates marked with Picard Tools (v2.4.1), reads split and trimmed and mapping qualities reassigned with GATK (v3.6) using methods SplitNCigarReads and ReassignOneMappingQuality, respectively. Variants were identified using GATK HaplotypeCaller and NTRK3 variants were inspected for previously reported resistance mutations. NTRK3 SNPs were observed at a maximum read depth of $\sim 450$ reads. Fusions were identified using arriba (v1.1.0). 


\section{TRK Immunohistochemistry}

Immunohistochemistry staining for TRK $A, B$, and $C$ expression was performed on paraffinembedded sections using the BenchMark ULTRA system (Roche) using the pan-TRK monoclonal antibody clone EPR17341 (Roche/Ventana).

\section{SPECC1L-NTRK2 Fusion Cloning}

Patient 2 RNA was reverse transcribed into cDNA using the SuperScript III First-Strand Synthesis System (Thermo Fisher Scientific). Truncated SPECC1L-NTRK2 (T SPECC1LNTRK2) was amplified from patient CDNA using SPECC1L Exon4 kozac F forward primer and NTRK R reverse primer (see Supplemental Table 2). Full-length SPECC1L-NTRK2 (FL SPECC1L-NTRK2) was amplified from patient CDNA using SPECC1L FL F forward primer and NTRK $R$ reverse primer (Supplemental Table 2). Fusions were PCR amplified using GoTaq Long PCR Master Mix (Promega) as per manufacturer's instructions. Truncated and full length SPECC1L-NTRK2 fusions were cloned into the pFTRE tight mTAad GFP (pFTRE GFP) lentiviral vector using the EcoRI restriction site. Fusion sequences were confirmed by Sanger sequencing (Australian Genome Research Facility).

\section{Cell Culture and Retroviral Transduction and Infection}

For lentiviral transduction, HEK293T cells were transfected using Effectene reagent (QIAGEN), using pCMV-dR8.2 dvpr packaging vector and pMD2.G VSV-G envelope. Ba/ F3 cells were transduced with lentivirus encoding pFTRE GFP (control construct), T SPECC1L-NTRK2, and FL SPECC1L-NTRK2 by spin infection and Polybrene reagent (Sigma-Aldrich). GFP-positive (fusion-expressing) Ba/F3 cells were sorted by flow cytometry following transduction. Cell lines were maintained in RPMI 1640 medium (Sigma-Aldrich) supplemented with $10 \%$ fetal bovine serum (FBS, Thermo Fisher Scientific) and $0.5 \mathrm{ng} / \mathrm{mL}$ recombinant murine IL-3 (Peprotech), at $37^{\circ} \mathrm{C}$ and $5 \% \mathrm{CO}_{2}$. For induction, $1 \mu \mathrm{g} / \mathrm{mL}$ of doxycycline was added to cultures, and fusion-expressing cells were maintained in RPMI supplemented with $10 \%$ FBS following selection.

\section{Western Blotting}

Western blot analysis was performed with antibodies targeting pan-TRK (EPR17341, Abcam) and $\beta$-actin (Sigma-Aldrich), as per manufacturer's instructions. HRP-conjugated secondary antibodies (GE Healthcare) were used, and a signal was detected using Luminata Forte Western HRP substrate (Millipore) and ChemiDoc XRS+ Imaging System (Bio-Rad Laboratories).

\section{Trypan Blue Cell Count Assay}

Cell lines were washed three times in phosphate-buffered saline (PBS) to remove residual IL3 from maintenance culture. Washed cells were then counted and seeded at $10^{5}$ live cells $/ \mathrm{mL}$ in 24-well plates in RPMI supplemented with $10 \% \mathrm{FBS}$ and $1 \mu \mathrm{g} / \mathrm{mL}$ doxycycline, with and without IL-3 $0.5 \mathrm{ng} / \mathrm{ml}$, and incubated at $37^{\circ} \mathrm{C}$ and $5 \% \mathrm{CO}_{2}$ for $10 \mathrm{~d}$. Live cells were counted by trypan blue exclusion, using TC20 Automated Cell Counter (Bio-Rad Laboratories) at 0, 3 , 5,7 , and $10 \mathrm{~d}$. Cells were maintained between $0.1-2 \times 10^{6} \mathrm{cell} / \mathrm{s} / \mathrm{mL}$ and were diluted at each timepoint to prevent overgrowth. Data were analyzed and visualized using GraphPad Prism v7.0 Software (GraphPad Software, Inc). Experiment was performed on two biologically independent lines in two independent technical replicates $(n=4)$. All data are presented as mean \pm SEM. 


\section{Drug Treatment Assay}

Cell lines were washed in PBS, counted, and seeded at $10^{4}$ live cells in 96-well plates in the presence (pFTRE empty vector control) or absence of $0.5 \mathrm{ng} / \mathrm{ml} \mathrm{IL-3}$, and $1 \mu \mathrm{g} / \mathrm{mL}$ doxycycline. Cells were treated with $0.0024-10 \mu \mathrm{M}$ dose range of imatinib or larotrectinib (Selleck Chemicals) or vehicle control (dimethylsulfoxide, DMSO, Sigma-Aldrich). Drug treatment plates were incubated at $37^{\circ} \mathrm{C}$ and $5 \% \mathrm{CO}_{2}$ for $48 \mathrm{~h}$. Propidium iodide (PI) exclusion was measured using an LSR X-20 Fortessa and high-throughput sequencer (HTS) (BD Biosciences). Data were analyzed using FlowJo v10.4 (FlowJo LLC) and GraphPad Prism v7.0 Software. Experiment was performed on two biologically independent lines in two independent technical replicates $(n=4)$. All data are presented as mean \pm SEM.

\section{ADDITIONAL INFORMATION}

\section{Data Deposition and Access}

The primary sequence data related to these cases are available through the European Genome-phenome Archive (https://www.ebi.ac.uk/ega/home) under the study ID EGASO0 001004679.

\section{Ethics Statement}

The study was approved by the Hunter New England Human Research Ethics Committee (Australia) with full written consent obtained from the parents, providing consent for the patients.

\section{Acknowledgments}

Tumor samples were supplied by the Children's Cancer Centre Tissue Bank at the Murdoch Children's Research Institute and The Royal Children's Hospital (www.mcri.edu.au/ childrenscancercentretissuebank). Establishment and running of the Children's Cancer Centre Tissue Bank is made possible through generous support by Cancer In Kids @ RCH (www.cika.org.au), the Royal Children's Hospital Foundation, and the Murdoch Children's Research Institute.

\section{Author Contributions}

D.-A.K.-Q. and P.G.E. conceived, designed and coordinated the study. L.M.B., A.S.-O., D.-A.K.-Q., and P.G.E. performed the in vitro experiments and analyzed data. M.W., C.M., A.K., M.P., S.B.F., M.J.C., and P.G.E. performed bioinformatics analysis. A.J.G. and M.R. reviewed the histology and performed the IHC. A.J.G. and L.E.L. contributed materials. D.-A.K.-Q., S.N., L.L., D.S.Z., J.R.H., and G.M.M. provided clinical data. J.D. and M.H. lead the precision medicine programs that provided the sequencing. D.-A.K.-Q., L.M.B., and P.G.E. wrote the original draft. All authors contributed to the written manuscript and approved the final manuscript.

\section{Funding}

iPredict is a Flagship project of Melbourne Genomics and funded by the State Government of Victoria (Department of Health and Human Services) and the 10 member organizations of the Melbourne Genomics Health Alliance. S.B.F. acknowledges the support of the National Health and Medical Research Council (NHMRC) Grant Funding APP1079329. P.G.E. acknowledges the support of NHMRC Project Grant APP1140626, the Samuel Nissen Charitable Foundation, and the Steven Walter Children's Cancer Foundation. G.M.M. acknowledges the support of Hyundai $4 \mathrm{~K}$ and Steven Walter Children's Cancer Foundation.
Received July 22, 2020; accepted in revised form October 22, 2020.
Competing Interest Statement The authors have declared no competing interest.

Khuong-Quang et al. 2020 Cold Spring Harb Mol Case Stud 6: a005710 
Zero is a national study of precision medicine in pediatric cancer supported by the Australian Federal Government Department of Health, New South Wales State Government, the Australian Cancer Research Foundation (ACRF), The Kids Cancer Alliance, Cancer Therapeutics Cooperative Research Centre (CTx CRC), Tour de Cure (tumor biobank personnel), Lions Kids Cancer Genome Project (WGS and personnel), Cure Brain Cancer Foundation, The Kids Cancer Project, University of New South Wales, Australian Genomics Health Alliance, Luminesce Alliance, The Medical Research Future Fund (MRFF) Australian Brain Cancer Mission, Minderoo Foundation's Eliminate Cancer Initiative, Zero Childhood Cancer Capacity Campaign, The Kinghorn Foundation, the Cancer Institute NSW, and NSW Health (Fellowship funding for M.J.C.). Cancer Australia provided personnel and computational support (APP1165556 to M.J.C.). Zero Childhood Cancer is a joint initiative led by Children's Cancer Institute and Sydney Children's Hospital Randwick.

\section{REFERENCES}

Atiq MA, Davis JL, Hornick JL, Dickson BC, Fletcher CDM, Fletcher JA, Folpe AL, Mariño-Enríquez A. 2020. Mesenchymal tumors of the gastrointestinal tract with NTRK rearrangements: a clinicopathological, immunophenotypic, and molecular study of eight cases, emphasizing their distinction from gastrointestinal stromal tumor (gist). Mod Pathol doi:10.1038/s41379-020-0623-z

Bongarzone I, Pierotti MA, Monzini N, Mondellini P, Manenti G, Donghi R, Pilotti S, Grieco M, Santoro M, Fusco A, et al. 1989. High frequency of activation of tyrosine kinase oncogenes in human papillary thyroid carcinoma. Oncogene 4: 1457-1462.

Bourgeois JM, Knezevich SR, Mathers JA, Sorensen PH. 2000. Molecular detection of the ETV6-NTRK3 gene fusion differentiates congenital fibrosarcoma from other childhood spindle cell tumors. Am J Surg Pathol 24: 937-946. doi:10.1097/00000478-200007000-00005

Cameron DL, Baber J, Shale C, Papenfuss AT, Valle-Inclan JE, Besselink N, Cuppen E, Priestley P. 2019. GRIDSS, PURPLE, LINX: unscrambling the tumor genome via integrated analysis of structural variation and copy number. bioRxiv doi:10.1101/781013

Cancer Genome Atlas Research Network. 2014. Integrated genomic characterization of papillary thyroid carcinoma. Cell 159: 676-690. doi:10.1016/j.cell.2014.09.050

Capper D, Jones DTW, Sill M, Hovestadt V, Schrimpf D, Sturm D, Koelsche C, Sahm F, Chavez L, Reuss DE, et al. 2018. DNA methylation-based classification of central nervous system tumours. Nature 555: 469474. doi:10.1038/nature26000

Cingolani P, Platts A, Wang le L, Coon M, Nguyen T, Wang L, Land SJ, Lu X, Ruden DM. 2012. A program for annotating and predicting the effects of single nucleotide polymorphisms, snpeff: SNPs in the genome of Drosophila melanogaster strain $w^{1118}$; iso-2; iso-3. Fly (Austin) 6: 80-92. doi:10.4161/fly.19695

Clarke M, Mackay A, Ismer B, Pickles JC, Tatevossian RG, Newman S, Bale TA, Stoler I, Izquierdo E, Temelso S, et al. 2020. Infant high grade gliomas comprise multiple subgroups characterized by novel targetable gene fusions and favorable outcomes. Cancer Discov 10: 942-963. doi:10.1158/2159-8290.CD-19-1030

Cocco E, Scaltriti M, Drilon A. 2018. NTRK fusion-positive cancers and TRK inhibitor therapy. Nat Rev Clin Oncol 15: 731-747. doi:10.1038/s41571-018-0113-0

Davis JL, Lockwood CM, Albert CM, Tsuchiya K, Hawkins DS, Rudzinski ER. 2018. Infantile NTRK-associated mesenchymal tumors. Pediatr Dev Pathol 21: 68-78. doi:10.1177/1093526617712639

Dickson BC, Swanson D, Charames GS, Fletcher CD, Hornick JL. 2018. Epithelioid fibrous histiocytoma: molecular characterization of ALK fusion partners in 23 cases. Mod Pathol 31: 753-762. doi:10.1038/mod pathol.2017.191

Drilon A, Laetsch TW, Kummar S, DuBois SG, Lassen UN, Demetri GD, Nathenson M, Doebele RC, Farago AF, Pappo AS, et al. 2018. Efficacy of larotrectinib in TRK fusion-positive cancers in adults and children. N Engl J Med 378: 731-739. doi:10.1056/NEJMoa1714448

Fouladi M, Gururangan S, Moghrabi A, Phillips P, Gronewold L, Wallace D, Sanford RA, Gajjar A, Kun LE, Heideman R. 2009. Carboplatin-based primary chemotherapy for infants and young children with CNS tumors. Cancer 115: 3243-3253. doi:10.1002/cncr.24362

Gatalica Z, Xiu J, Swensen J, Vranic S. 2019. Molecular characterization of cancers with NTRK gene fusions. Mod Pathol 32: 147-153. doi:10.1038/s41379-018-0118-3

Gayevskiy V, Roscioli T, Dinger ME, Cowley MJ. 2019. Seave: a comprehensive web platform for storing and interrogating human genomic variation. Bioinformatics 35: 122-125. doi:10.1093/bioinformatics/bty540 
Guerreiro Stucklin AS, Ryall S, Fukuoka K, Zapotocky M, Lassaletta A, Li C, Bridge T, Kim B, Arnoldo A, Kowalski $\mathrm{PE}$, et al. 2019. Alterations in ALK/ROS1/NTRK/MET drive a group of infantile hemispheric gliomas. Nat Commun 10: 4343. doi:10.1038/s41467-019-12187-5

Hodgson A, Pun C, Djordjevic B, Turashvili G. 2020. NTRK-rearranged cervical sarcoma: expanding the clinicopathologic spectrum. Int J Gynecol Pathol doi:10.1097/PGP.0000000000000669.

Hsiao SJ, Zehir A, Sireci AN, Aisner DL. 2019. Detection of tumor NTRK gene fusions to identify patients who may benefit from tyrosine kinase (TRK) inhibitor therapy. J Mol Diagn 21: 553-571. doi:10.1016/j.jmoldx .2019.03.008

Hung YP, Fletcher CDM, Hornick JL. 2018. Evaluation of pan-TRK immunohistochemistry in infantile fibrosarcoma, lipofibromatosis-like neural tumour and histological mimics. Histopathology 73: 634-644. doi:10 $.1111 /$ his.13666

Jennings LJ, Arcila ME, Corless C, Kamel-Reid S, Lubin IM, Pfeifer J, Temple-Smolkin RL, Voelkerding KV, Nikiforova MN. 2017. Guidelines for validation of next-generation sequencing-based oncology panels: a joint consensus recommendation of the Association for Molecular Pathology and College of American Pathologists. J Mol Diagn 19: 341-365. doi:10.1016/j.jmoldx.2017.01.011

Knezevich SR, McFadden DE, Tao W, Lim JF, Sorensen PH. 1998. A novel ETV6-NTRK3 gene fusion in congenital fibrosarcoma. Nat Genet 18: 184-187. doi:10.1038/ng0298-184

Laetsch TW, DuBois SG, Mascarenhas L, Turpin B, Federman N, Albert CM, Nagasubramanian R, Davis JL, Rudzinski E, Feraco AM, et al. 2018. Larotrectinib for paediatric solid tumours harbouring NTRK gene fusions: phase 1 results from a multicentre, open-label, phase 1/2 study. Lancet Oncol 19: 705-714. doi:10 .1016/S1470-2045(18)30119-0

$\mathrm{Li} \mathrm{H}$, Durbin R. 2009. Fast and accurate short read alignment with Burrows-Wheeler transform. Bioinformatics 25: 1754-1760. doi:10.1093/bioinformatics/btp324

Mackay A, Burford A, Carvalho D, Izquierdo E, Fazal-Salom J, Taylor KR, Bjerke L, Clarke M, Vinci M, Nandhabalan M, et al. 2017. Integrated molecular meta-analysis of 1,000 pediatric high-grade and diffuse intrinsic pontine glioma. Cancer Cell 32: 520-537 e525. doi:10.1016/j.ccell.2017.08.017

Marchiò C, Scaltriti M, Ladanyi M, lafrate AJ, Bibeau F, Dietel M, Hechtman JF, Troiani T, López-Rios F, Douillard JY, et al. 2019. ESMO recommendations on the standard methods to detect NTRK fusions in daily practice and clinical research. Ann Oncol 30: 1417-1427. doi:10.1093/annonc/mdz204

Martin-Zanca D, Hughes SH, Barbacid M. 1986. A human oncogene formed by the fusion of truncated tropomyosin and protein tyrosine kinase sequences. Nature 319: 743-748. doi:10.1038/319743a0

McLaren W, Gil L, Hunt SE, Riat HS, Ritchie GR, Thormann A, Flicek P, Cunningham F. 2016. The Ensembl variant effect predictor. Genome Biol 17: 122. doi:10.1186/s13059-016-0974-4

McWhirter JR, Galasso DL, Wang JY. 1993. A coiled-coil oligomerization domain of Bcr is essential for the transforming function of Bcr-Abl oncoproteins. Mol Cell Biol 13: 7587-7595. doi:10.1128/MCB.13.12.7587

Murphy DA, Ely HA, Shoemaker R, Boomer A, Culver BP, Hoskins I, Haimes JD, Walters RD, Fernandez D, Stahl JA, et al. 2017. Detecting gene rearrangements in patient populations through a 2-step diagnostic test comprised of rapid IHC enrichment followed by sensitive next-generation sequencing. Appl Immunohistochem Mol Morphol 25: 513-523. doi:10.1097/PAl.0000000000000360

Nelson AW, Schrock AB, Pavlick DC, Ali SM, Atkinson EC, Chachoua A. 2019. Novel SPECC1L-MET fusion detected in circulating tumor DNA in a patient with lung adenocarcinoma following treatment with erlotinib and osimertinib. J Thorac Oncol 14: e27-e29. doi:10.1016/j.jtho.2018.10.160

Paila U, Chapman BA, Kirchner R, Quinlan AR. 2013. Gemini: integrative exploration of genetic variation and genome annotations. PLoS Comput Biol 9: e1003153. doi:10.1371/journal.pcbi.1003153

Pavlick D, Schrock AB, Malicki D, Stephens PJ, Kuo DJ, Ahn H, Turpin B, Allen JM, Rosenzweig M, Badizadegan K, et al. 2017. Identification of NTRK fusions in pediatric mesenchymal tumors. Pediatr Blood Cancer 64. doi:10.1002/pbc.26433

Penault-Llorca F, Rudzinski ER, Sepulveda AR. 2019. Testing algorithm for identification of patients with TRK fusion cancer. J Clin Pathol 72: 460-467. doi:10.1136/jclinpath-2018-205679

Pinese M, Lacaze P, Rath EM, Stone A, Brion MJ, Ameur A, Nagpal S, Puttick C, Husson S, Degrave D, et al. 2020. The medical genome reference bank contains whole genome and phenotype data of 2570 healthy elderly. Nat Commun 11: 435. doi:10.1038/s41467-019-14079-0

Rabban JT, Devine WP, Sangoi AR, Poder L, Alvarez E, Davis JL, Rudzinski E, Garg K, Bean GR. 2020. NTRK fusion cervical sarcoma: a report of three cases, emphasising morphological and immunohistochemical distinction from other uterine sarcomas, including adenosarcoma. Histopathology 77: 100-111. doi:10.1111/ his.14069

Roberts KG, Li Y, Payne-Turner D, Harvey RC, Yang YL, Pei D, McCastlain K, Ding L, Lu C, Song G, et al. 2014. Targetable kinase-activating lesions in Ph-like acute lymphoblastic leukemia. N Engl J Med 371: 10051015. doi:10.1056/NEJMoa1403088

Rodrigues GA, Park M. 1993. Dimerization mediated through a leucine zipper activates the oncogenic potential of the MET receptor tyrosine kinase. Mol Cell Biol 13: 6711-6722. doi:10.1128/MCB.13.11.6711 
Saadi I, Alkuraya FS, Gisselbrecht SS, Goessling W, Cavallesco R, Turbe-Doan A, Petrin AL, Harris J, Siddiqui U, Grix AW, et al. 2011. Deficiency of the cytoskeletal protein SPECC1L leads to oblique facial clefting. Am J Hum Genet 89: 44-55. doi:10.1016/j.ajhg.2011.05.023

Sahm F, Schrimpf D, Jones DT, Meyer J, Kratz A, Reuss D, Capper D, Koelsche C, Korshunov A, Wiestler B, et al. 2016. Next-generation sequencing in routine brain tumor diagnostics enables an integrated diagnosis and identifies actionable targets. Acta Neuropathol 131: 903-910. doi:10.1007/s00401-015-1519-8

Saunders CT, Wong WS, Swamy S, Becq J, Murray LJ, Cheetham RK. 2012. Strelka: accurate somatic smallvariant calling from sequenced tumor-normal sample pairs. Bioinformatics 28: 1811-1817. doi:10.1093/ bioinformatics/bts271

Seager M, Aisner DL, Davies KD. 2019. Oncogenic gene fusion detection using anchored multiplex polymerase chain reaction followed by next generation sequencing. J Vis Exp doi:10.3791/59895

Shen MM. 2013. Chromoplexy: a new category of complex rearrangements in the cancer genome. Cancer Cell 23: 567-569. doi:10.1016/j.ccr.2013.04.025

Sheng WQ, Hisaoka M, Okamoto S, Tanaka A, Meis-Kindblom JM, Kindblom LG, Ishida T, Nojima T, Hashimoto H. 2001. Congenital-infantile fibrosarcoma. A clinicopathologic study of 10 cases and molecular detection of the ETV6-NTRK3 fusion transcripts using paraffin-embedded tissues. Am J Clin Pathol 115: 348-355. doi:10.1309/3H24-E7T7-V37G-AKKQ

Stransky N, Cerami E, Schalm S, Kim JL, Lengauer C. 2014. The landscape of kinase fusions in cancer. Nat Commun 5: 4846. doi:10.1038/ncomms5846

Surrey LF, Jain P, Zhang B, Straka J, Zhao X, Harding BN, Resnick AC, Storm PB, Buccoliero AM, Genitori L, et al. 2019. Genomic analysis of dysembryoplastic neuroepithelial tumor spectrum reveals a diversity of molecular alterations dysregulating the MAPK and PI3K/MTOR pathways. J Neuropathol Exp Neurol 78: 1100-1111. doi:10.1093/jnen/nlz101

Torre M, Vasudevaraja V, Serrano J, DeLorenzo M, Malinowski S, Blandin AF, Pages M, Ligon AH, Dong F, Meredith DM, et al. 2020. Molecular and clinicopathologic features of gliomas harboring NTRK fusions. Acta Neuropathol Commun 8: 107. doi:10.1186/s40478-020-00980-z

Van der Auwera GA, Carneiro MO, Hartl C, Poplin R, Del Angel G, Levy-Moonshine A, Jordan T, Shakir K, Roazen D, Thibault J, et al. 2013. From FASTQ data to high confidence variant calls: the genome analysis toolkit best practices pipeline. Curr Protoc Bioinformatics 43: 11.10.11-11.10.33. doi:10.1002/ 0471250953.bi1110s43

Wong M, Mayoh C, Lau LMS, Khuong-Quang DA, Pinese M, Kumar A, Barahona P, Wilkie EE, Sullivan P, Bowen-James R, et al. 2020. Whole genome, transcriptome and methylome profiling enhances actionable target discovery in high-risk pediatric cancer. Nat Med doi: 10.1038/s41591-020-1072-4.

Zhou X, Edmonson MN, Wilkinson MR, Patel A, Wu G, Liu Y, Li Y, Zhang Z, Rusch MC, Parker M, et al. 2016. Exploring genomic alteration in pediatric cancer using ProteinPaint. Nat Genet 48: 4-6. doi:10 $.1038 / \mathrm{ng} .3466$ 


\section{COLD SPRING HARBOR Molecular Case Studies}

\section{Recurrent SPECC1L-NTRK fusions in pediatric sarcoma and brain tumors}

Dong-Anh Khuong-Quang, Lauren M. Brown, Marie Wong, et al.

Cold Spring Harb Mol Case Stud 2020, 6: a005710 originally published online November 3, 2020

Access the most recent version at doi: $10.1101 / \mathrm{mcs} .0005710$
Supplementary http://molecularcasestudies.cshlp.org/content/suppl/2020/11/12/mcs.a005710.D Material C1

References This article cites 49 articles, 5 of which can be accessed free at: http://molecularcasestudies.cshlp.org/content/6/6/a005710.full.html\#ref-list-1

License This article is distributed under the terms of the Creative Commons Attribution-NonCommercial License, which permits reuse and redistribution, except for commercial purposes, provided that the original author and source are credited.

Email Alerting Receive free email alerts when new articles cite this article - sign up in the box at the Service top right corner of the article or click here. 